\title{
Les coraux scléractiniaires du Burdigalien supérieur du Bassin de Bonifacio (Corse-du-Sud)
}

Christian CHAIX

Département Histoire de la Terre, Muséum national d'Histoire naturelle, Centre de Recherche sur la Paléobiodiversité et les Paléoenvironnements (CR2P: CNRS, MNHN, UPMC-Paris 06; Sorbonne Universités), case postale 38, 57 rue Cuvier, F-75231 Paris cedex 05 (France)

scleractiniaire@live.fr

Françoise GALLONI

$\mathrm{Cz}$ N. Assas, La géologie appliquée, 7 avenue de Toulon, F-13006 Marseille (France)

fgalloni@yahoo.fr

Michelle FERRANDINI

F-20250 Casanova de Venaco (France) ferrandini.jean@wanadoo.fr

Jean-Jacques CORNÉE

Université Montpellier 2, CNRS UMR 5243 Géosciences Montpellier, place E. Bataillon, F-34095 Montpellier Cedex 05 (France) jean-jacques.cornee@gm.univ-montp2.fr

Publié le 25 septembre 2015

MOTS CLÉS Récifs coralliens,

Corse du Sud, Burdigalien supérieur, Associations coralliennes,

Paléoenvironnements.

Chaix C., Galloni F., Ferrandini M. \& Cornée J.-J. 2015. - Les coraux scléractiniaires du Burdigalien supérieur du Bassin de Bonifacio (Corse-du-Sud). Geodiversitas 37 (3): 367-381. http://dx.doi.org/10.5252/g2015n3a5

\section{RÉSUMÉ}

Au Burdigalien supérieur, des récifs coralliens se sont développés dans la Formation de Cala di Labra, aux alentours de Bonifacio (Corse-du-Sud, France). Ils sont organisés en trois épisodes successifs R1, $\mathrm{R} 2$ et R3. La faune corallienne comprenant 16 espèces récifales, est décrite pour la première fois. Deux associations coralliennes sont distinguées. Les principaux genres constructeurs sont Porites Link, 1807, Tarbellastraea Alloiteau, 1950 et Thegioastraea Sismonda, 1871. La diversité corallienne est maximale avec 15 espèces ( 8 genres) dans l'épisode R1, puis réduite (4 espèces appartenant à 3 genres) pour l'épisode R2, l'épisode R3 renfermant des coraux mal préservés pour être identifiés. Les formes de croissance sont en grande majorité massives étalées et superposées, associées à des conditions paléoenvironnementales de haute à moyenne énergie (rides de courant, bioclastes usés, peu de formes branchues), sous apports terrigènes réguliers. Les récifs, pour la plupart frangeants et à zonation récifale discrète, se sont développés en domaine de plate-forme peu profonde. Cette étude reprend également toutes les citations d'espèces dans la littérature pour cette région et replace ces épisodes coralliens remarquables dans l'évolution plus générale du Bassin Liguro-Provençal. 


\begin{abstract}
The Upper Burdigalian scleractinian corals from Bonifacio (South Corsica).

In Upper Burdigalian time, coral reefs developed within the Cala di Labra Formation, in the Bonifacio area (South Corsica, France). They are organized into three successive coral reef episodes R1, R2 and R3. The coral fauna comprising 16 reefal species, is described in detail for the first time. Two coral associations are observed. The major framebuilders are Porites Link, 1807, Tarbellastraea Alloiteau, 1950 and Thegioastraea Sismonda, 1871. The coral diversity is maximum (15 species, 8 genera) during R1, then lower during R2 ( 4 species, 3 genera). Corals of the R3 episode are poorly preserved, so unable for systematic identification. The coral growth forms are mostly tabular massive. The colonies lived under high to moderate energy conditions (ripple-marks, abraded bioclasts, few branching colonies), and permanent siliciclastic inputs. Buildups are mainly fringing reefs with discrete vertical zonation, deposited into shallow water platform domain. This study also synthesizes the coral species previously mentioned in the regional literature and replaces these remarkable coral reefs within the evolution of the Ligurian-Provençal Basin.

\author{
KEY WORDS \\ Coral reefs, \\ South Corsica, \\ Bonifacio
Upper Burdigalian, \\ Coral associations,
}

Palaeoenvironments.
\end{abstract}

\section{INTRODUCTION ET HISTORIQUE}

Les faunes coralliennes miocènes d'Atlantique (Cahuzac \& Chaix 1996, 2009), de Téthys, de Paratéthys et du Moyen Orient ont fait l'objet d'une synthèse (Bosellini \& Perrin 2008; Perrin \& Bosellini 2012). À partir des réajustements biostratigraphiques des séries sédimentaires, la répartition des coraux à zooxanthelles a suivi les étapes géodynamiques et l'évolution paléogéographique qui ont prévalues au cours du Miocène.

Pour le Burdigalien, les édifices construits composés d'assemblages coralliens à diversité modérée (1 à 15 genres au total) sont recensés dans les domaines ouest-téthysien (Mallorca et bloc corso-sarde), est-téthysien (Turquie, Iran, 17 espèces/15 genres) et au Moyen Orient (références et synthèse in Buchbinder 1996; Esteban 1996; Bassant 1999; Bassant et al. 2005; références et cartes paléogéographiques de Popov et al. 2004 modifiées in Perrin \& Bosellini 2012; Harzhauser et al. 2007). La distribution des coraux a ensuite connu dans ces zones une expansion latitudinale au Langhien, pendant un optimum climatique. Les édifices construits (15 espèces/10-12 genres) étaient alors plus répandus dans les régions circum-méditerranéennes (Grenade, Catalogne, Languedoc, Algérie), dans le Golfe de Suez et en Mer Rouge (Perrin \& Bosellini 2012).

Dans le Bassin Liguro-Provençal, en Corse et en Sardaigne, Pedley (1996) et Perrin \& Bosellini (2012), signalent des faunes coralliennes composant quelques biohermes au Burdigalien et au Langhien.

Toutefois, dans le détroit des Bouches de Bonifacio (Fig. 1A, B), sur les marges d'un haut fond granitique entre la Corse et la Sardaigne, une activité récifale construite était bien implantée principalement au Burdigalien supérieur. Dans un premier temps, les études régionales stratigraphiques des bassins miocènes de Corse et plus particulièrement du Sud de la Corse (Orszag-Sperber \& Pilot 1976; Orszag-Sperber 1978; Ferrandini et al. 1998, 2003; Loÿe-Pilot \& Magné 1989; Caron \& Loÿe-Pilot 1990; Loÿe-Pilot et al. 2004; Arnaud et al. 1989; Monleau et al.
1996) et du Nord de la Sardaigne (Assorgia et al. 1988; Barca \& Spano 2002; Mazzei \& Oggiano 1990; Arnaud et al. 1992; Martini et al. 1992; Cherchi et al. 2008) ont fait état des récifs coralliens burdigaliens sans détailler leur contenu. En Corse cependant, quelques espèces du Miocène étaient mentionnées dans les premiers travaux sur la systématique des scléractiniaires (Michelin 18401847 ; d'Orbigny 1852; Milne-Edwards \& Haime 1857). Les recherches du XIX siècle (Locard 1873; Locard \& Cotteau 1877; Peron 1886) ont été citées par Chevalier (1961). Elles ont permis d'identifier sept espèces, auxquelles Chevalier a ajouté trois autres, mais de localités inconnues. Plus récemment, des études axées sur le contenu faunique des récifs de Bonifacio et leurs processus d'édification (Ferrandini et al. 2002, 2003, 2010; Galloni 2003; Galloni et al. 2001, 2014; Galloni \& Cornée 2014; Orsini et al. sous presse) et de Santa Teresa di Gallura (Brandano et al. 2010 ; Tomassetti \& Brandano 2013; Tomassetti et al. 2013) ont mis en évidence leurs similitudes biosédimentologiques et paléoenvironnementales.

Cet article a pour objectifs de décrire la composition de la faune corallienne burdigalienne de Bonifacio, et de discuter des facteurs paléoenvironnementaux liés à son développement.

\section{ORGANISATION GÉNÉRALE DES DÉPÔTS ÉTUDIÉS}

Entre 1997 et 2010, plusieurs missions de terrain menées par une équipe pluridisciplinaire ont affiné les cadres biostratigraphique, lithostratigraphique, biosédimentologique et géodynamique des dépôts miocènes de Bonifacio (Fig. 1A, B). L'étude de la plate-forme mixte carbonatée récifale du Burdigalien supérieur (Formation de Cala di Labra) a permis de détailler les paléoenvironnements récifaux et leurs modalités d'édification (Ferrandini et al. 2002, 2003, 2010; Galloni 2003; Galloni et al. 2001, 2014; Galloni \& Cornée 2014; Orsini et al. sous presse). Les récifs se répartissent en 


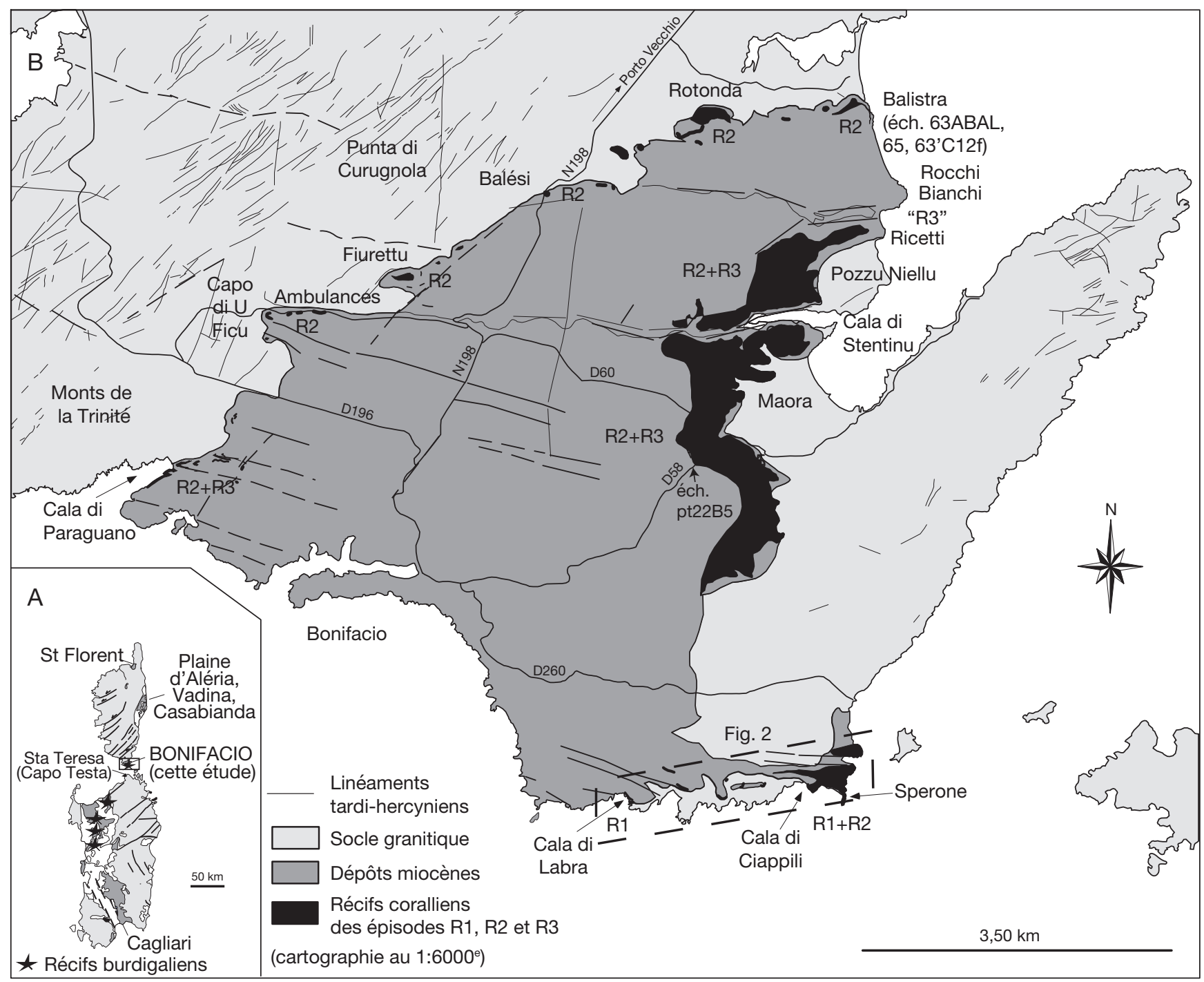

FIG. 1. - A, Récifs burdigaliens dans le bloc corso-sarde, d'après Carmignani et al. (1995) ; B, carte de localisation des principaux affleurements récifaux aux alentours de Bonifacio (Corse-du-Sud), d'après Galloni (2003).

trois épisodes successifs (R1, R2A/B et R3) datés Burdigalien supérieur par les microfaunes, en particulier les Miogypsinidae (Ferrandini et al. 2002, 2003, 2010 ; Galloni 2003; Galloni et al. 2001, 2014; Orsini et al. sous presse). Frangeants et côtiers, ils ne possèdent pas de zonation verticale marquée (Galloni 2003; Galloni \& Cornée 2014). Ils se sont mis en place suivant une géométrie rétrogradante sur le socle hercynien admettant plusieurs paléoreliefs. Les épisodes coralliens ont souligné les paléorivages successifs lors de la transgression burdigalienne. Ils sont alignés suivant une direction globale NE-SO à la bordure septentrionale de la plate-forme et une direction NS à sa bordure orientale (Fig. 1B). Les principaux sites sont Cala di Labra, Cala di Ciappili, Sperone, Maora-intersection D58/D60, Cala di Stentinu, Balistra, Rotonda, Balési, Fiurettu, Ambulances, Capo di U Ficu et Cala di Paraguano. Les trois épisodes récifaux se sont installés à la fin de l'ouverture rapide du bassin liguro-provençal (Gattacecca et al. 2007; Orsini et al. sous presse; Oudet et al. 2010).

\section{MATÉRIEL ET MÉTHODES}

Les récifs de Bonifacio ont été analysés à l'échelle des corps unitaires composant chaque épisode récifal (Galloni 2003; Galloni \& Cornée 2014) : inventaire des formes de croissance, des espèces et des processus biosédimentologiques et cartographie récifale des principaux affleurements. Plus d'une soixantaine d'échantillons ont été récoltés sur les récifs des épisodes R1 et R2 (Fig. 2; Tableau 1). L'identification des coraux a pu être réalisée principalement sur les macrostructures car, dans de nombreux cas, les spécimens présentent des recristallisations (sparite en mosaïque). Les colonies très altérées des récifs des épisodes $\mathrm{R} 2 \mathrm{~B}$ et $\mathrm{R} 3$ n'ont pas pu faire l'objet d'une étude exhaustive. De ce fait, le nombre d'espèces recensées est susceptible d'être revu à la hausse aux niveaux qualitatif et quantitatif. Les échantillons sont déposés dans la Collection de Paléontologie du Muséum national d'Histoire naturelle de Paris (MNHN.F). Les observations réalisées sont complétées par les spécimens issus des collections his- 
TABLEAU 1. - Inventaire des coraux scléractiniaires dans les récifs R1 et R2 du Burdigalien supérieur aux environs de Bonifacio (Sud de la Corse). Abréviation: $\mathbf{N}$, nombre total de spécimens.

\begin{tabular}{|c|c|c|c|c|c|c|c|c|}
\hline \multicolumn{4}{|c|}{$\begin{array}{l}\text { Numéro d'échantillon sur les coupes lithologiques/ } \\
\text { localisation géographique (Galloni 2003) }\end{array}$} & \multirow[b]{2}{*}{$\begin{array}{l}\text { Numérotation } \\
\text { MNHN.F }\end{array}$} & \multirow[b]{2}{*}{ Espèce } & \multirow[b]{2}{*}{$\mathbf{N}$} & \multirow[b]{2}{*}{$\begin{array}{l}\text { Type de } \\
\text { colonie }\end{array}$} & \multirow[b]{2}{*}{ Figures } \\
\hline $\begin{array}{l}\text { R1 Cala di } \\
\text { Labra }\end{array}$ & $\begin{array}{c}\text { R1 Sperone- } \\
\text { Ciappili - } \\
\text { coupes } \\
\text { C6, C6bis }\end{array}$ & $\begin{array}{l}\text { R2 Sperone- } \\
\text { Ciappili } \\
\text { coupe C7 }\end{array}$ & $\begin{array}{l}\text { R2 autres } \\
\text { localités } \\
\text { (cf. Fig. 1) }\end{array}$ & & & & & \\
\hline CLR 1,7 & & & & MNHN.F.A51127 & Stylophora affinis & 1 & plocoïde & $3 A, B$ \\
\hline \multirow[t]{2}{*}{ CLR base } & & & & MNHN.F.A51128 & Favia cf. valceppii & 1 & massive plocoïde & $3 C, D$ \\
\hline & & & & MNHN.F.A51156 & $\begin{array}{l}\text { Favites cf. neglecta } \\
\text { (Ghisoni) }\end{array}$ & & massive cérioïde & $3 \mathrm{E}$ \\
\hline \multirow[t]{2}{*}{ CLR 1,7} & & & & MNHN.F.A51129 & Favites neglecta & 9 & massive cérioïde & $3 F$ \\
\hline & SP21R1bis & & & MNHN.F.A51130 & Favites detecta & 2 & massive cérioïde & $4 \mathrm{~A}$ \\
\hline \multirow[t]{3}{*}{ CLR 1, 7} & SP21R1bis & & & MNHN.F.A51131 & $\begin{array}{l}\text { Heliastraea } \\
\text { (Aquitanastraea) } \\
\text { incrustans }\end{array}$ & 12 & massive plocoïde & 4B \\
\hline & SP21R1bis & & & MNHN.F.A51132 & Heliastraea s.l. sp. 1 & 1 & massive plocoïde & $4 \mathrm{C}$ \\
\hline & SP21R1bis & & & & Heliastraea s.I. sp. 2 & 1 & massive plocoïde & \\
\hline \multirow[t]{3}{*}{ Cala di Labra } & & & & MNHN.F.A51134 & $\begin{array}{l}\text { Tarbellastraea } \\
\text { carryensis major }\end{array}$ & 1 & massive plocoïde & $4 \mathrm{E}$ \\
\hline & & 33bis & & MNHN.F.A51135 & $\begin{array}{l}\text { Thegioastraea } \\
\text { diversiformis }\end{array}$ & 3 & $\begin{array}{l}\text { massive, } \\
\text { plocoïde à } \\
\text { subcérioïde }\end{array}$ & $4 \mathrm{~F}$ \\
\hline & SP21R1bis & & & MNHN.F.R63843 & $\begin{array}{l}\text { Thegioastraea corsica } \\
\text { n. comb. }\end{array}$ & 2 & massive plocoïde & $5 A, B$ \\
\hline $\begin{array}{l}\text { CLR } 1,7 \\
\text { CLOY }\end{array}$ & & & & $\begin{array}{l}\text { MNHN.F.A51136, } \\
\text { MNHN.F.A51137 }\end{array}$ & $\begin{array}{l}\text { Thegioastraea } \\
\text { speciosa }\end{array}$ & 3 & massive plocoïde & $5 C, D$ \\
\hline \multirow[t]{2}{*}{$\begin{array}{l}\text { Cala di } \\
\text { Labra }\end{array}$} & Sperone & & & MNHN.F.A51138 & $\begin{array}{l}\text { Thegioastraea } \\
\text { burdigalensis }\end{array}$ & 2 & massive plocoïde & $5 \mathrm{E}$ \\
\hline & & 37 & & & Thegioastraea indét. & fragments & massive plocoïde & \\
\hline Cala di Labra & & & & & $\begin{array}{l}\text { Plesiastraea } \\
\text { (Paleoplesiastraea) } \\
\text { desmoulinsi }\end{array}$ & 1 & massive plocoïde & \\
\hline $7^{\prime}$ & $41 b$ & 37 & & & Porites sp. & 9 & massive cérioïde & \\
\hline
\end{tabular}

toriques de Michelin, d'Orbigny et Chevalier. La répartition géographique dans le domaine téthysien et les dénominations des niveaux récifaux historiques sont précisées, en fonction du cadre stratigraphique et des réajustements biostratigraphiques récents. Les spécimens récoltés ont fait l'objet de révisions systématiques permettant d'identifier 13 espèces et de laisser trois autres en nomenclature ouverte.

\section{ÉTUDE SYSTÉMATIQUE}

Cette étude repose sur la systématique traditionnelle des scléractiniaires, et n'intègre pas les nouvelles techniques morphométriques, microstructurales et anatomiques, entre autres moléculaires. Les fossiles, dont les parties molles ne sont pas conservées, ont leurs éléments squelettiques fréquemment érodés ou recristallisés. De plus, cette nouvelle systématique ne nous semble pas adaptée à des différenciations d'espèces et genres pour lesquels la systématique traditionnelle permet de distinguer des taxons sans ambiguïté possible.
Famille STYLOPHORIDAE Milne-Edwards et Haime, 1857

Stylophora affinis Duncan, 1863

(Fig. 3A, B)

Stylophora affinis Duncan, 1863: 436, pl. XVI, fig. 4. — Vaughan 1919: 336. - Cahuzac \& Chaix 1996: 112, 117, 119.

Matériel type. - “Coll. Geol. Soc.” fide Duncan (1863).

MATÉRIEL EXAMINÉ. — 1 exemplaire de Cala di Labra (MNHN.F.A51127).

NIVEAU TYPE. — Nivajè shale.

LOCALITÉ TYPE. - Saint Domingue; pas de localité précise désignée.

RÉPARTITION STRATIGRAPHIQUE ET GÉOGRAPHIQUE. - Miocène inférieur et moyen des Antilles (Duncan 1863; Vaughan 1919).

Chattien, Aquitanien et Burdigalien d'Aquitaine (Cahuzac \& Chaix 1996).

Burdigalien supérieur de Corse (secteur sud-est: 1er épisode récifal de Cala di Labra-CLR 1, 7). 


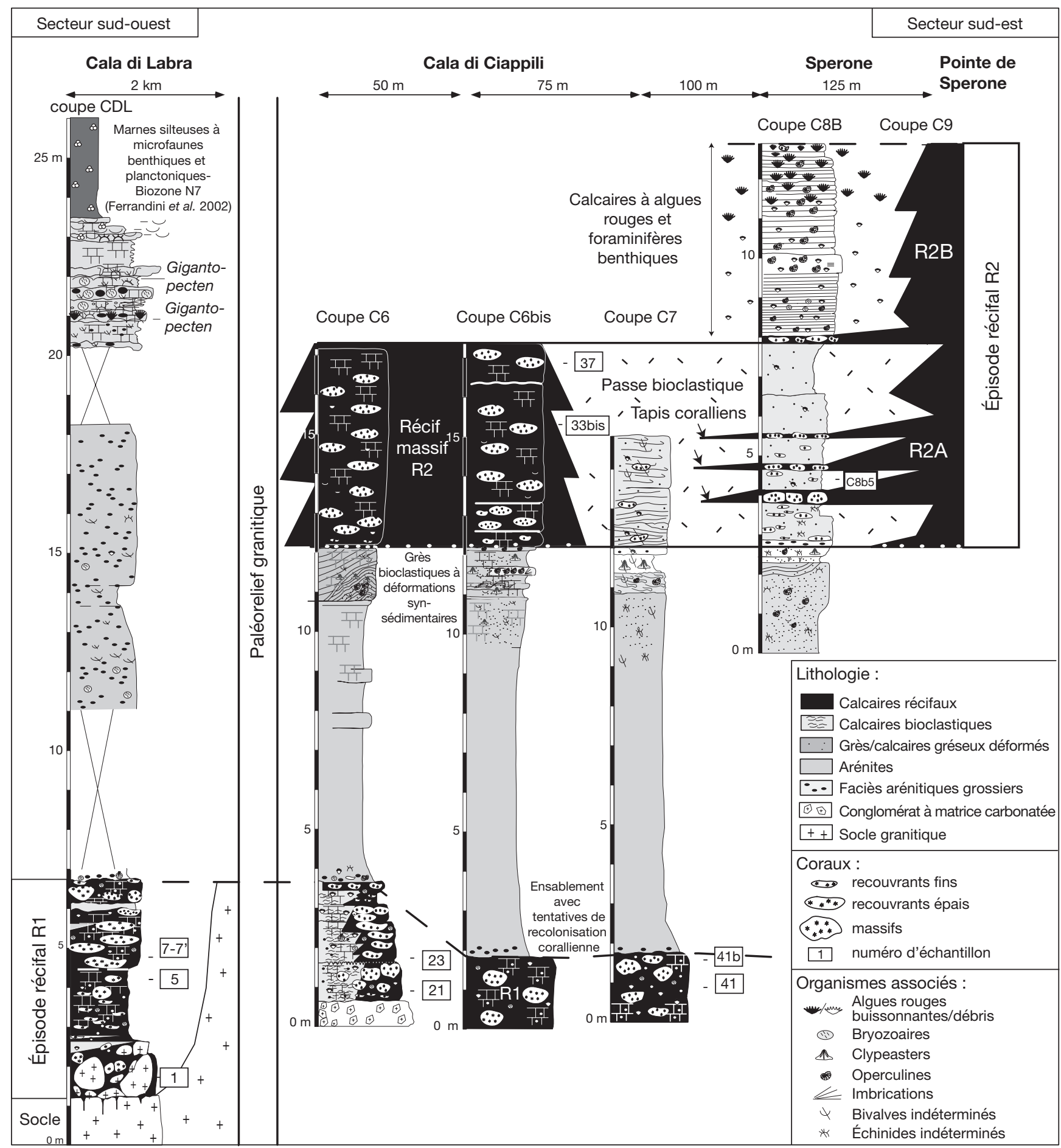

FIG. 2. - Position des échantillons dans les récifs des épisodes R1 et R2 entre Cala di Labra et Sperone (description des coupes, Galloni 2003).

\section{DESCRIPTION}

Colonies à disposition plocoïde des calices (calices de forme arrondie, plus ou moins espacés les-uns des autres) et columelle constituée par la jonction des septes, parfois styliforme. Le squelette est imperforé; chez cette espèce, les calices ne renferment que six septes, le diamètre calicinal n'excède jamais $0,9 \mathrm{~mm}$ et les distances entre centres calicinaux sont de 1,8 $\mathrm{mm}$ au maximum; une faible ligne en relief est parfois visible entre les calices, caractéristique de cette espèce. Notre exemplaire est un négatif. 


\section{Famille FAVIIDAE Gregory, 1900}

Nos exemplaires ne sont pas suffisamment bien préservés pour les attribuer à une famille sur une base moléculaire. Par contre ils s'insèrent sans problème dans la famille des Faviidae, qui doit être préservée.

Favia cf. valceppii Zuffardi-Commerci, 1932 (Fig. 3C, D)

Favia valceppii Zuffardi-Commerci, 1932: 123, pl. XV, fig. 10.

MatéRIEL TYPe. - Holotype par monotypie, coll. Cantamessa.

Matériel examiné. - 1 exemplaire de Cala di Labra (MNHN.F.A51128)

NiveAU TYPE. — Miocène moyen.

LOCALITÉ TYPE. - Colline de Turin, sans précisions supplémentaires.

RÉPARTITION STRATIGRAPHIQUE ET GÉOGRAPHIQUE. - Burdigalien supérieur de Corse (secteur sud-est: 1er épisode récifal de Cala di Labra-CLR base).

Miocène moyen de Val Ceppi (colline de Turin, Italie) (ZuffardiCommerci 1932).

\section{DESCRIPTION}

Colonie massive, plocoïde, à bourgeonnement intracalicinal et division incomplète des calices, caractéristique du genre Favia Milne Edwards, 1857 ; columelle spongieuse; côtes fines. Dentition distale non préservée. Le nombre de septes par calice (trois cycles ou un peu plus, soit 24 à 28 septes) et le diamètre calicinal maximal $(15 \mathrm{~mm})$ sont caractéristiques de cette espèce.

Espèce très rare, connue jusqu'ici seulement par deux exemplaires: l'holotype italien et notre exemplaire corse, ce qui justifie le doute quant à l'identification.

\section{Favites neglecta (Michelotti in d'Achiardi, 1868)} (Fig. 3E, F)

Aphrastraea? neglecta Michelotti in d'Achiardi, 1868: 13, pl. I, fig. 10-11.

Favites neglecta - Chevalier 1961: 138, pl. X, fig. 2, XVII, fig. 16 et tfs 45a, b. - Cahuzac \& Chaix 1996: 112, 117, 119.

Matériel type. - Collection d'Achiardi.

Matériel examiné. - Un exemplaire de "Ghisoni» (MNHN.F.A51156; environs de Corte, Corse) (coll. Michelin); 9 exemplaires de Cala di Labra (MNHN.F.A51129).

NiveAU TYPE. - Miocène moyen.

LOCAlité TYPe. - Colline de Turin.

RÉPARTITION STRATIGRAPHIQUE ET GÉOGRAPHIQUE. — Un exemplaire de Corse ("Ghisoni» selon ancienne étiquette) identifié comme douteux par Chevalier in coll. Michelin (MNHN) (Chevalier 1961). Après examen de l'exemplaire, nous sommes du même avis.
Chattien, Aquitanien et Burdigalien d'Aquitaine (Cahuzac \& Chaix 1996) et de la Nerthe (Chevalier 1961).

Burdigalien supérieur de Corse (secteur sud-est: $1{ }^{\text {er }}$ épisode récifal de Cala di Labra-CLR 1, 7), Burdigalien de Turquie (Chevalier 1961). Miocène moyen de la Colline de Turin (Michelotti 1838), du Languedoc, de Catalogne (Chevalier 1961).

\section{DESCRIPTION}

Espèce très commune dans tout l'Oligo-Miocène atlanticoméditerranéen, sauf au Miocène supérieur. La colonie est massive, cérioïde (calices jointifs, donc non circulaires), à petite columelle spongieuse; les dissépiments sont très abondants. Cette espèce se distingue des espèces voisines par son nombre de septes (quatre cycles incomplets, soit un nombre de septes variable entre 24 et 48 ) et son diamètre calicinal maximal $(13 \mathrm{~mm})$.

\section{Favites detecta (Michelotti in Sismonda, 1871)} (Fig. 4A)

Septastraea detecta Michelotti in Sismonda, 1871:314, pl. VIII, fig. 6.

Favites detecta - Cahuzac \& Chaix 1996: 112.

MATÉRIEL TYPE. - Collection Michelotti.

Matériel examiné. - 2 exemplaires de Sperone-Ciappili (MNHN.F.A51130).

NIVEAU TYPE. — Miocène moyen.

LOCALITÉ TYPE. - Turin.

RÉPARTITION STRATIGRAPHIQUE ET GÉOGRAPHIQUE. - Chattien d'Aquitaine (Cahuzac \& Chaix 1996).

Aquitanien et Burdigalien d'Aquitaine (identifications récentes).

Burdigalien supérieur de Corse (secteur sud-est: 1er épisode récifal de Sperone-Ciappili - SP21R1bis).

Miocène moyen de la colline de Turin (Sismonda 1871).

\section{DESCRIPTION}

Comme l'avait observé Chevalier (1961) cette espèce est très voisine de la précédente; elle ne s'en distingue que par ses calices plus petits, qui ne dépassent jamais $10 \mathrm{~mm}$ de diamètre. Espèce relativement commune, que nous avons retrouvée dans l'Aquitanien et le Burdigalien d'Aquitaine, postérieurement à notre publication de 1996.

\section{Heliastraea (Aquitanastraea) incrustans (Osasco, 1897)} (Fig. 4B)

Heliastraea incrustans Osasco, 1897: 8, fig. 3.

Aquitanastraea incrustans - Chevalier 1961: 187.

Heliastraea (Aquitanastraea) incrustans - Cahuzac \& Chaix 1996: 112, 119, 121.

Matériel type. — «Abondant" (fide Osasco 1897); l'holotype provenant de la colline de Turin est perdu (fide Chevalier 1954: 152). 

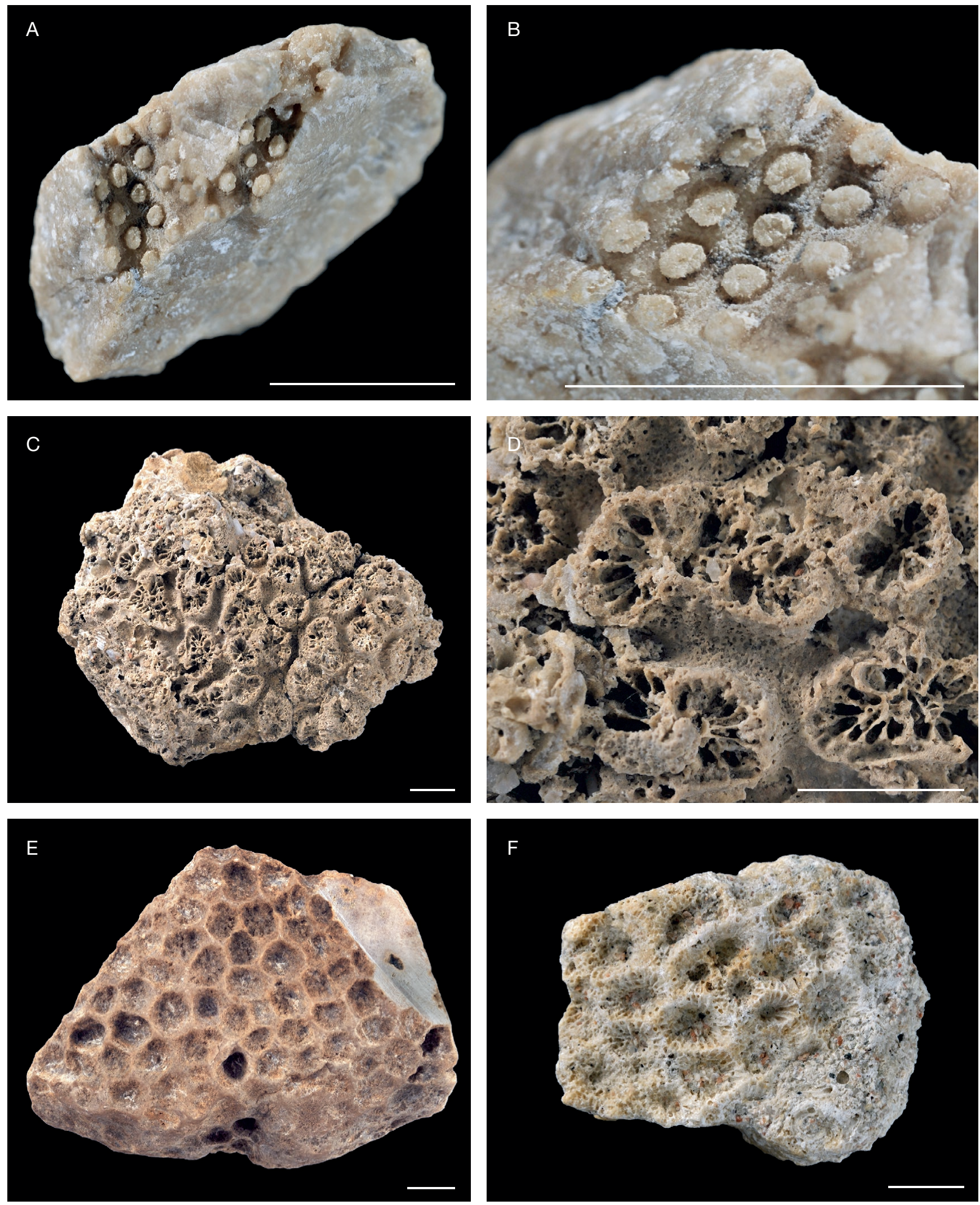

FIG. 3. - A, B, Stylophora affinis Duncan, 1863, MNHN.F.A51127; C, D, Favia cf. valceppii Zuffardi-Commerci, 1932, MNHN.F.A51128; E, Favites cf. neglecta (Michelotti in d'Achiardi, 1868), MNHN.F.A51156, spécimen de Ghisoni ; F, Favites neglecta, MNHN.F.A51129. Échelles: 10 mm. 
Matériel eXaminé. - Neuf exemplaires de Cala di Labra et trois exemplaires de Sperone-Ciappili (MNHN.F.A51131).

NiveAU TYPE. - Miocène moyen.

LOCALITÉ TYPe. - Colline de Turin.

RÉPARTITION STRATIGRAPHIQUE ET GÉOGRAPHIQUE. - Chattien, Aquitanien, Burdigalien et Langhien d'Aquitaine (Cahuzac \& Chaix 1996 + découvertes récentes). Cette espèce est commune en Aquitaine. Burdigalien supérieur de Corse (secteur sud-est: $1^{\text {er }}$ épisode récifal de Cala di Labra - CLR 1, 7; 1 er épisode récifal de Sperone-CiappiliSP21 R1bis).

Miocène moyen de la colline de Turin (Osasco 1897).

Deux exemplaires d' Heliastraea Milne-Edwards \& Haime, 1857 s.l. ont été récoltés à Sperone (1 er épisode récifal). Ils ne sont pas identifiables spécifiquement, mais au vu de leurs différences morphologiques, ils pourraient appartenir à deux espèces différentes, Heliastraea s.l. sp. 1 (Fig. 4C) et Heliastraea s.l. sp. 2.

\section{DESCRIPTION}

Les Heliastraea sont des coraux de morphologie massive, à disposition plocoïde des calices, et columelle spongieuse. Le sous-genre Aquitanastraea se distingue par son exothèque possédant des micropiliers verticaux qui joignent les dissépiments. Heliastraea (A.) incrustans est identifiée par ses calices possédant quatre cycles incomplets de costo-septes (entre 24 et 48), un diamètre calicinal maximal de $5 \mathrm{~mm}$, et une distance centre-à-centre de $7 \mathrm{~mm}$.

Tarbellastraea reussiana (Milne-Edwards \& Haime, 1850) (Fig. 4D)

La synonymie, la description et la répartition stratigraphique et géographique ont été détaillées in Chaix \& Saint Martin (2008).

MATÉRIEL EXAMINÉ. - Dix exemplaires de Cala di Labra; 18 exemplaires de Sperone-Ciappili (MNHN.F.A51133).

RÉPARTITION STRATIGRAPHIQUE ET GÉOGRAPHIQUE. — Burdigalien supérieur de Corse (secteur sud-est: 1 $1^{\text {er }}$ épisode récifal de Cala di Labra -CLR 1, 7; 1er épisode récifal de Sperone-Ciappili - SP21 R1bis; 2e épisode récifal de Sperone-Ciappili - 33bis, 37). Autres localités citées par les auteurs: Burdigalien supérieur (Saint-Florent; Crovo); Tortonien (Casabianda, Aléria, Vadina).

\section{REMARQUES}

L'espèce Solenastraea peroni Locard, 1877 (Locard 1877; Peron 1886; Chevalier 1958) est signalée dans la littérature du Burdigalien corse. Il s'agit en fait de Tarbellastraea reussiana. Chevalier (1958) cite également Tarbellastraea ellisiana (préalablement nommée Heliastraea ellisi par Locard (1876) pour Vadina, en Plaine orientale, et Crovo, à Bonifacio), il s'agit très probablement là aussi de $T$. reussiana.

Tarbellastraea carryensis major Chevalier, 1961 (Fig. 4E)

Tarbellastraea carryana Chevalier, 1958: 115.
Tarbellastraea ellisiana Chevalier, 1958: 115.

Tarbellastraea carryensis major - Chevalier 1961: 198, pl. VII, fig. 8.

MATÉRIEL TYPE. - L'holotype provient de l'anse du Grand-Vallat (Nerthe); trois paratypes des environs de Carry-le-Rouet.

Matériel examiné. - Un exemplaire de Cala di Labra (MNHN.F.A51134).

NiveAu TYPE. - Aquitanien.

LOCALITÉ TYPE. — Anse du Grand-Vallat (Nerthe).

RÉPARTITION STRATIGRAPHIQUE ET GÉOGRAPHIQUE. - ChattienAquitanien de la Nerthe (Chevalier 1961; Oudet 2008; Oudet et al. 2010) : l'espèce est présente dans l'assise "C7" (récif R1) de Chevalier (1961). Tarbellastraea carryensis est observée sur la totalité des récifs provençaux (récif $\mathrm{R} 1$ à l'est et à la base de Cap de Nautes: assises "C3 à C11 "; récif R2 au Cap de Nautes: assises «C13 et C14»; Récif R3 entre la Pointe de Carry et la Tuilière: assise "F3»; R4 à la pointe de Carry: assise «F4»).

Burdigalien supérieur de Corse (secteur sud-est: 1er épisode récifal à Cala di Labra).

\section{DESCRIPTION}

Cette espèce, jusqu'ici connue uniquement de la Nerthe, se distingue facilement de T. reussiana: elle possède quatre cycles incomplets de costo-septes (T. reussiana: trois complets), la distance entre centres calicinaux peut atteindre $7,5 \mathrm{~mm}$ (T. reussiana: $4 \mathrm{~mm}$ ) et le diamètre calicinal peut atteindre $5 \mathrm{~mm}$ (T. reussiana: $3,5 \mathrm{~mm})$.

\section{Thegioastraea diversiformis (Michelin, 1842)} (Fig. 4F)

Astrea diversiformis Michelin, 1842: 59, pl. 12, fig. 5.

Thegioastraea diversiformis - Chevalier 1961: 220, pl. XX, fig. 5, tf. 74. — Cahuzac \& Chaix 1996: 112, 117, 119.

Matériel tYpe. - Collections Michelin et Michelotti.

Matériel examiné. - Trois exemplaires de Sperone-Ciappili (MNHN.F.A51135).

NIVEAU TYPE. — Miocène moyen.

LOCALITÉ TYPE. - Colline de Turin.

Répartition stratigraphique et Géographique. - Chattien, Aquitanien et Burdigalien d'Aquitaine (Michelin 1842, Chevalier 1961, Cahuzac \& Chaix 1996).

Aquitanien du Portugal et de la Nerthe (récif R3: niveau "F3 », Chevalier 1961).

Burdigalien de Provence (Chevalier 1961) et Burdigalien supérieur de Corse (Chevalier 1961: Bonifacio; récoltes Galloni dans le secteur sud-est: 2 ème épisode récifal de Sperone-Ciappili - 33bis). Miocène inférieur d'Algérie (Chevalier 1961).

Miocène moyen de la Loire (Chaix \& Cahuzac 2005), de la colline de Turin (Michelin 1842; Chevalier 1961) et de Grèce (Chevalier 1961).

\section{DESCRIPTION}

Les Thegioastraea Sismonda, 1871, abondants dans l'OligoMiocène atlantico-méditerranéen, forment de grosses colonies massives à grands calices disposés de manière plocoïde 

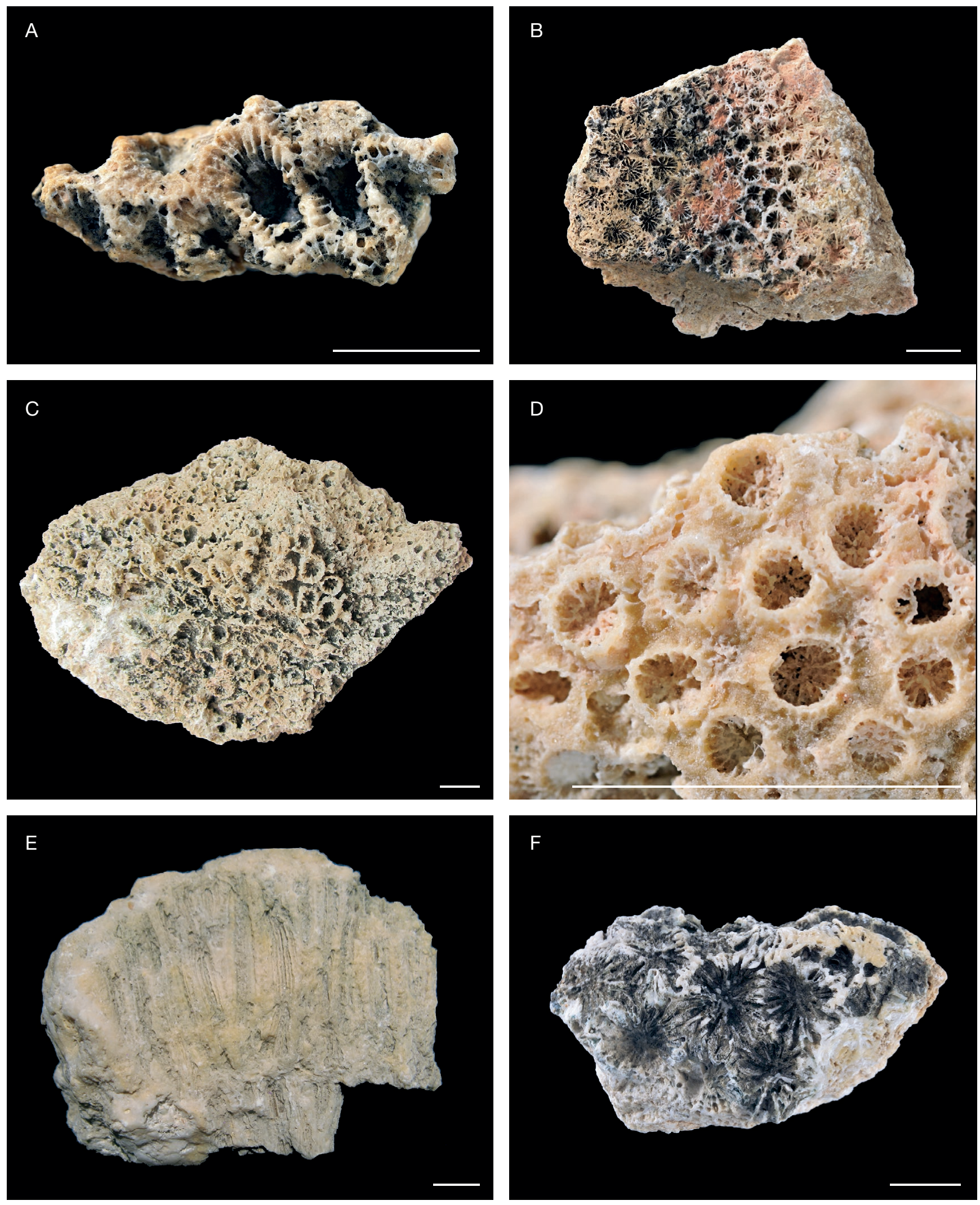

FIG. 4. - A, Favites detecta (Michelotti in Sismonda, 1871), MNHN.F.A51130; B, Heliastraea (Aquitanastraea) incrustans (Osasco, 1897), MNHN.F.A51131; C, Heliastraea s.I. sp. 1, MNHN.F.A51132; D, Tarbellastraea reussiana (Milne-Edwards \& Haime, 1850), MNHN.F.A51133; E, Tarbellastraea carryensis major Chevalier, 1961, MNHN.F.A51134; F, Thegioastraea diversiformis (Michelin, 1842), MNHN.F.A51135. Échelles: 10 mm. 
ou subcérioïde, à costo-septes subconfluents, sans muraille, et grosse columelle spongieuse. Le squelette est dense et peut faire penser aux Mussidae, cependant la dentition distale des costo-septes est beaucoup moins épineuse. Thegioastraea diversiformis se caractérise par quatre cycles incomplets d'éléments radiaires et un diamètre calicinal atteignant $15 \mathrm{~mm}$.

Thegioastraea corsica (d'Orbigny, 1852) n. comb.

(Fig. 5A, B)

Astrea corsica d'Orbigny, 1852: vol. III, 147, n²746”'.

Heliastraea Haimei d'Archiac in Tchihatcheff, 1869: Paléontologie, atlas, pl. XV.

Thegioastraea cf. speciosa-Chevalier (in coll.), n. syn.

Thegioastraea diversiformis - Chevalier 1961: 220 (partie corse du matériel étudié), n. syn.

MATÉRIEL TYPE. — Syntype MNHN.F.R63843 (typothèque MNHN).

MatéRIel eXAminé. — Deux exemplaires de Sperone-Ciappili.

NiveAu TYPE. — Miocène.

LOCALITÉ TYPE. - Corse sans précision.

RÉPARTITION STRATIGRAPHIQUE ET GÉOGRAPHIQUE. - Miocène inférieur de Turquie (d'Archiac 1869).

Burdigalien supérieur de la région de Bonifacio (Chevalier 1961): de par sa gangue rosée, l'exemplaire de d'Orbigny semble de même provenance. Récoltes Galloni: secteur sud-est (Sperone-Ciappili: 1er épisode récifal - SP 21 R1bis).

\section{DESCRIPTION}

Cette espèce était uniquement connue par le syntype de d'Orbigny. Elle possède tous les caractères du genre Thegioastraea décrits plus haut, et trois cycles complets d'éléments radiaires, ou un peu plus, ce qui l'apparente à l'espèce T. speciosa. Son diamètre calicinal (jusqu'à $16 \mathrm{~mm}$ ) l'en différencie très facilement.

Ayant retrouvé l'exemplaire unique de Astrea corsica dans la typothèque du MNHN (syntype MNHN.F.R63843), nous rangeons donc cette espèce dans le genre Thegioastraea, et mettons dans sa synonymie les deux exemplaires proposés par Chevalier, pour la Corse, en $T$. cf. speciosa et T. diversiformis, pour les raisons indiquées ci-dessus.

\section{Thegioastraea speciosa (Sismonda, 1871)}

$$
\text { (Fig. 5C, D) }
$$

Goniastraea speciosa Sismonda, 1871: 319, pl. IV, fig. 6.

Thegioastraea cf. speciosa-Chevalier 1961: 215.

MATÉRIEL TYPE. — Aucun renseignement de l'auteur sur le matériel étudié ni sur son lieu de rangement géographique.
Matériel eXaminé. - Trois exemplaires de Cala di Labra (MNHN.F.A51136, A51137).

NIVEAU TYPE. — Miocène moyen.

Localité TYPE. - Colline de Turin.

RÉPARTITION STRATIGRAPHIQUE ET GÉOGRAPHIQUE. - Burdigalien supérieur de Corse (secteur sud-est: 1er épisode récifal de Cala di Labra - CLR 1, 7, CLOY).

Miocène moyen de la colline de Turin (Sismonda 1871) et du Languedoc (Chevalier 1961).

\section{DESCRIPTION}

Espèce assez rare, connue jusqu'ici par quelques exemplaires seulement, dont certains douteux. Cette espèce se caractérise par son faible nombre de costo-septes (trois cycles ou un peu plus) et son petit diamètre calicinal $(6 \mathrm{~mm})$; les centres calicinaux sont distants de $7 \mathrm{~mm}$. C'est le cas des autres exemplaires attribués à cette espèce par Chevalier.

\section{Thegioastraea burdigalensis Chevalier, 1961}

(Fig. 5E)

Thegioastraea burdigalensis Chevalier, 1961: 221, pl. VIII, fig. 3, 21, tf. 75. — Cahuzac \& Chaix 1996: 119.

MatéRIEl tYPe. - Holotype (le Peloua, Gironde); matériel non retrouvé.

MatéRIEl EXAMINÉ. - Un exemplaire de Sperone, un exemplaire de Cala di Labra (MNHN.F.A51138), deux topotypes.

NivEAU TYPE. - Burdigalien.

LOCALITÉ TYPe. — Le Peloua (Gironde).

RÉPARTITION STRATIGRAPHIQUE ET GÉOGRAPHIQUE. — Burdigalien d'Aquitaine (Chevalier 1961; Cahuzac \& Chaix 1996).

Burdigalien supérieur de Corse (Chevalier 1961: 1 exemplaire de Bonifacio - coll. Peron; récoltes Galloni: 1er épisode récifal à Cala di Labra et Sperone).

Miocène inférieur d'Algérie (Chevalier 1961).

Miocène moyen de la colline de Turin (Chevalier 1961).

Des fragments de Thegioastraea indéterminables spécifiquement ont été trouvés dans le 2 ème épisode récifal de Sperone-Ciappili (37).

\section{DESCRIPTION}

Espèce très commune au Burdigalien, elle se caractérise par quatre cycles incomplets de septes et un diamètre calicinal maximal de $12 \mathrm{~mm}$.

\section{Plesiastraea (Paleoplesiastraea) desmoulinsi}

Milne-Edwards \& Haime, 1851

Cette espèce, très fréquente dans le Miocène supérieur méditerranéen, a été décrite en détail in Chaix \& Saint Martin (2008); on s'y reportera.

Matériel eXAminé. - En Corse, elle ne se trouve que dans le Burdigalien supérieur à Cala di Labra (1 er épisode récifal). Chevalier (1961) en a trouvé un exemplaire douteux dans la collection Peron (MNHN). 

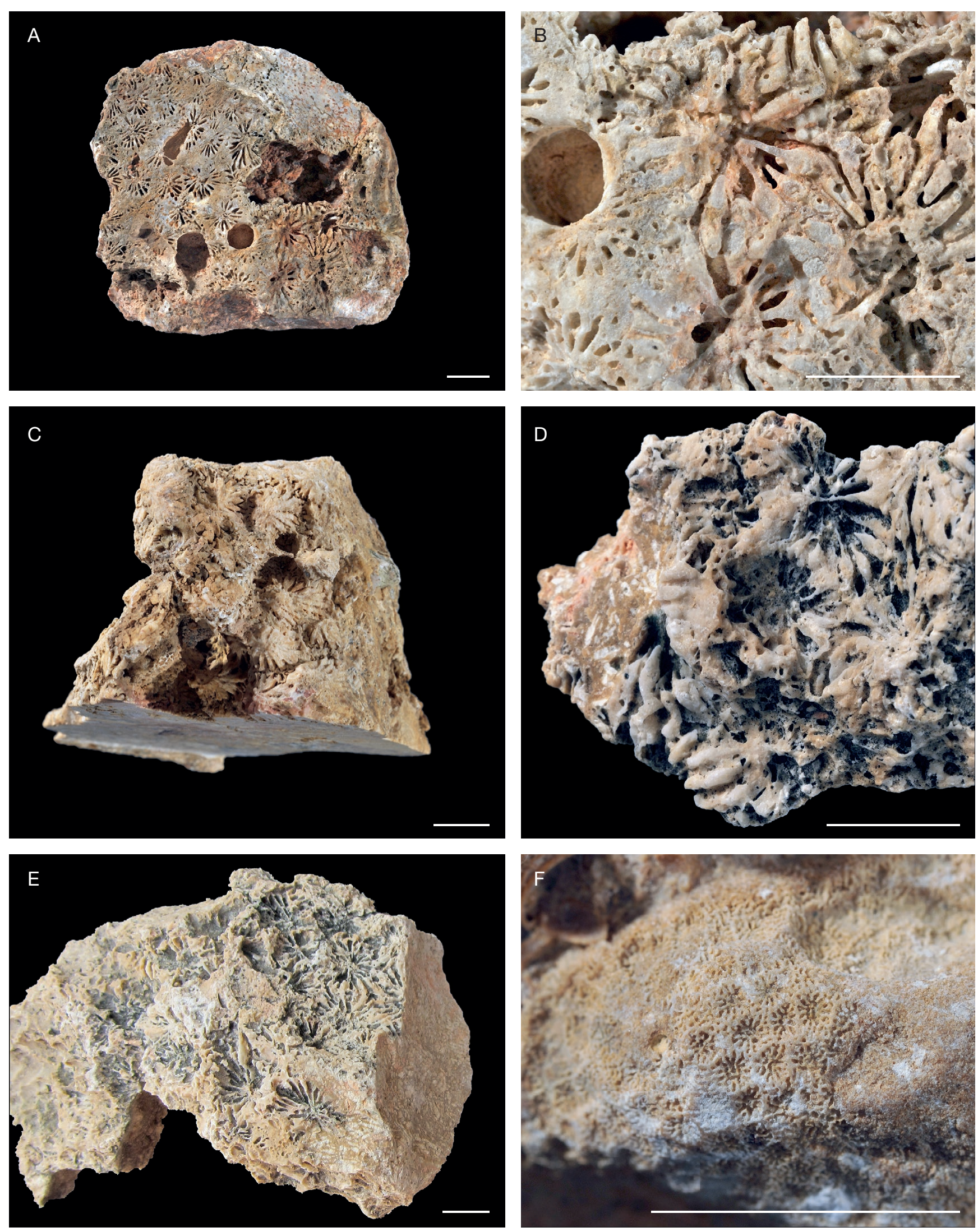

FIG. 5. - A, B, Thegioastraea corsica (d'Orbigny, 1852) n. comb., MNHN.F.R63843; C, Thegioastraea cf. speciosa, MNHN.F.A51136; D, Th. speciosa (Sismonda, 1871), MNHN.F.A51137; E, Thegioastraea burdigalensis Chevalier, 1961, MNHN.F.A51138; F, Porites arenosa (Esper, 1797), MNHN.F.A51139. Échelles: 10 mm. 
Famille PORITIDAE Gray, 1842

\section{Porites arenosa (Esper, 1797)}

(Fig. 5F)

On se reportera également pour cette espèce à la note de Chaix \& Saint Martin (2008).

\section{MATÉRIEL EXAMINÉ. — MNHN.F.A51139.}

RÉPARTITION STRATIGRAPHIQUE ET GÉOGRAPHIQUE. - Cette espèce semble très abondante dans le Burdigalien supérieur de Corse, d'après les récoltes de Françoise Galloni:

Secteur sud-est: Cala di Labra (1er épisode récifal) : CLR 1, 7 (6 exemplaires) ; 5 (1 ex.) ; Sperone-Ciappili (1er épisode récifal) : SP $21 \mathrm{R}$ 1 bis (1 ex.) ; 23 (1 ex.) ; 41 (1 ex.). 2ème épisode récifal: 37 (4 ex.). Secteur nord-est: 6 ex. répartis dans tous les points de fouilles (2ème épisode récifal: PT 22 B5 à l'intersection D58/D60, près de Maora - 63Abal, 65, 63’C 12 fà Balistra).

\section{DESCRIPTION}

Le Porites collegniana Michelin, 1842 cité par Chevalier (1958) est certainement un $P$. arenosa. Des Porites indéterminables spécifiquement ont été trouvés dans le 1 er épisode récifal de Cala di Labra (7’ -2 ex.) et de Sperone-Ciappili ( $41 b-3$ ex.), ainsi que dans le 2 ème épisode récifal de Sperone-Ciappili $(37-4$ ex.).

En plus de toutes ces espèces, Chevalier (1961) créé Acanthastraea termofurae Chevalier, 1961 (1 ex. de Bonifacio de la collection Peron), que nous n'avons pas retrouvée. Cette espèce, si le spécimen est usé, peut être difficile à distinguer d'un Thegioastraea.

\section{RÉSULTATS/DISCUSSION}

\section{ASSOCIATIONS CORALLIENNES ET ÉVOLUTION PALÉO-} ENVIRONNEMENTALE AU COURS DU BURDIGALIEN SUPÉRIEUR Les genres coralliens décrits à Bonifacio sont: Favia Oken, 1815, Favites Link, 1807, Tarbellastraea Alloiteau, 1950, Porites, Heliastraea (Aquitanastraea), Thegioastraea, Stylophora Schweigger, 1819 et Plesiastraea (Paleoplesiastraea) Chevalier, 1961. Porites, Tarbellastraea et Thegioastraea sont les plus fréquents. Les 13 espèces identifiées, en plus des trois espèces en nomenclature ouverte, appartiennent uniquement aux Poritidae et Favidae. Plusieurs familles sont absentes (Acroporidae, Siderastraeidae, Agaricidae, Turbinariidae, Mussidae) alors qu'elles sont représentées dans les faciès récifaux et subrécifaux burdigaliens de Méditerranée (Chevalier 1961; Budd et al. 1996) ou d'Aquitaine (Cahuzac \& Chaix 1996). La diversité corallienne à Bonifacio est très modérée, représentative des assemblages téthysiens s.l. au Burdigalien supérieur (Chevalier 1961; Esteban 1996; Perrin \& Bosellini 2012).

L'ensemble de la faune étudiée, composant deux associations coralliennes distinctes, traduit des conditions locales d'eau claire, chaude, bien oxygénée, de turbidité marquée par des apports détritiques fréquents en provenance du socle, empêchant les Porites d'être dominants. La fraction inorganique dans les matrices récifales, estimée en lames minces à partir d'une charte visuelle, ainsi que les données paléoécologiques et paléoenvironnementales proposées sont issues des travaux de Ferrandini et al. (2002, 2003, 2010), Galloni (2003), Galloni et al. (2001, 2014), et Galloni \& Cornée (2014).

La première association typique de l'épisode R1 est la plus diversifiée (15 taxons, 8 genres): Stylophora affinis, Favia cf. valceppii, Favites neglecta et $F$. detecta, Heliastraea (3 espèces), Tarbellastraea reussiana et $T$. carryensis major, Thegioastraea (3 espèces), Plesiastraea (Paleoplesiastraea) desmoulinsi, Porites arenosa et Porites sp. Les récifs de cet épisode sont frangeants, soumis à un facteur hydrodynamique élevé et à des apports siliciclastiques grossiers réguliers (15-20\% de la matrice; Galloni \& Cornée 2014). Les coraux sont lamellaires fins, recouvrants épais, en choux-fleurs, empilés les uns sur les autres. Ils sont associés à des foraminifères benthiques (amphistégines, miogypsines), des bryozoaires, des bivalves et des algues rouges et sont fréquemment perforés par des organismes lithophages. Tous les genres ont des représentants actuels, exceptés Tarbellastraea, Heliastraea (Aquitanastraea) et Thegioastraea, et se rencontrent (révision des espèces actuelles de Veron 2000) dans des zones très peu profondes, voire intertidales battues par les vagues (Favia, Stylophora). Un épisode d'ensablement généralisé a interrompu leur croissance. Cette association corallienne caractériserait des paléoprofondeurs de l'ordre de 5-15 m, estimation appuyée par l'étude des microfaunes associées (Ferrandini et al. 2002).

La deuxième association caractérisant l'épisode R2 est appauvrie (quatre espèces, trois genres) : Thegioastraea diversiformis, Tarbellastraea reussiana, Porites arenosa et Porites sp. Les récifs frangeants et côtiers se sont développés sur l'ensemble de la plate-forme (Ferrandini et al. 2002, 2003, 2010; Galloni et al. 2001, 2014; Galloni 2003; Galloni \& Cornée 2014; Orsini et al. sous presse). Les colonies sont recouvrantes épaisses et empilées, associées à des foraminifères benthiques (amphistégines, miogypsines, operculines), des algues rouges, des bivalves, des échinides et des bryozoaires (Ferrandini et al. 2002, 2003, 2010). Le détritisme est encore très présent (15-20\%). Certains récifs de mode battu à Ciappili-Sperone s'indentent dans une passe bioclastique de haute énergie, à tapis coralliens, colonies convexes et rides de courants (Galloni \& Cornée 2014; Galloni et al. 2014). À Paraguano, des colonies branchues non identifiables, en plus des colonies recouvrantes, se sont développées à la base du récif, en milieu plus protégé. Les paléoprofondeurs estimées sont de l'ordre de $5-30 \mathrm{~m}$.

La faible diversité corallienne au Burdigalien dans la région de Bonifacio est comparable à celle du Miocène supérieur de Méditerranée (Chaix \& Saint Martin 2008). De nombreux facteurs peuvent avoir contribué à cette chute de la diversité corallienne à partir de l'épisode R2, les genres disparus ne pouvant en effet vivre qu'en conditions récifales climax (température, courants, oxygénation, turbidité) pour le développement récifal. De probables compétitions intra-spécifiques (facteurs écologiques autogéniques) ont également pu intervenir. On peut penser ici à des conditions de mer plus franches observées dans les cortèges de microfaunes (Ferrandini et al. 2002). Les changements paléoenvironnementaux (turbidité forte, 
modifications climatiques, chute de la température des eaux, courants plus importants, apports en nutriments...) pourraient avoir conduit à appauvrir la faune corallienne réduite aux trois genres coralliens ubiquistes et plus résistants Porites, Thegioastraea et Tarbellastraea.

Ces derniers composent également les récifs de l'épisode R3 (Paraguano, Maora), en majorité sous forme de colonies recouvrantes épaisses. Ils sont associés à des calcaires à corallinacées buissonnantes au voisinage des reliefs granitiques, et accessoirement à des bryozoaires, des bivalves et des foraminifères benthiques (amphistégines, miogypsines). Les conditions d'énergie sont devenues plus modérées et le détritisme plus rare (5\%), en relation avec les variations du niveau marin. Les épisodes $\mathrm{R} 2 \mathrm{~B} / \mathrm{R} 3$ ont enregistré une phase d'approfondissement du milieu puis de comblement (Orsini et al. sous presse; Galloni et al. 2014; Galloni \& Cornée 2014).

Ainsi, à Bonifacio, la distribution des récifs ainsi que leur composition et leurs variations latérales ont été contrôlées par les paléoreliefs granitiques, la proximité des paléorivages, les côtes découpées, les variations du niveau marin relatif et les conditions paléoenvironnementales locales (Galloni et al. 2014). La disparition définitive de l'activité récifale corallienne est enregistrée dans les dépôts sus-jacents de plate-forme plus tempérée (Formation de Bonifacio, Ferrandini et al. 2003 ; André et al. 2011 ; Reynaud et al. 2013) traduisant à partir du Langhien inférieur, de profonds changements paléocourantologiques et paléogéographiques dans ce secteur.

\section{ÉQUIVALENTS RÉCIFAUX AU BURDIGALIEN SUPÉRIEUR}

Les récifs construits du Burdigalien supérieur de Bonifacio sont comparables à ceux de Sardaigne septentrionale de même âge (Fig. 1), notamment à Santa Teresa di Gallura (OrszagSperber 1978; Monleau et al. 1996; Ferrandini et al. 2003; Brandano et al. 2010; Orsini et al. sous presse; Galloni et al. 2014) : installation sur le socle hercynien, récifs de mode battu, coraux recouvrants dominants, apports siliciclastiques fréquents. Brandano et al. (2010) ont décrit 6 genres (Porites, Tarbellastraea, Montastraea, Thegioastraea, Favites et Siderastraea). Nous y avons récolté en complément des colonies recouvrantes de Porites sp., Heliastraea (Aquitanastraea) incrustans, Tarbellastraea reussiana et Thegioastraea sp. Les Bouches de Bonifacio ont formé une aire sédimentaire propice pour leur développement quasi permanent au Burdigalien supérieur. Les deux îles occupaient alors une position similaire à l'actuelle sur la bordure orientale du bassin liguro-provençal en cours d'ouverture (Gattacecca et al. 2007; Oudet et al. 2010). Cette activité récifale burdigalienne ne posséderait pas d'équivalent sur les marges sud-provençale (Chevalier 1961; Maurel-Ferrandini 1975; Demarcq \& Perriaux 1984; Oudet 2008), languedocienne et catalane (cf. références et réajustements biostratigraphiques in Perrin \& Bosellini 2012). Les récifs du Burdigalien supérieur-Langhien des îles Baléares, de Lisbonne et de la province Algarve seraient d'affinité atlantique (Cahuzac \& Chaix 1996); ceux du Nord de l'Afrique restent à ce jour peu documentés (Chevalier 1961; Perrin \& Bosellini 2012). Ce caractère unique est d'autant plus remarquable que dans les provinces coralliennes du Piémont (Chevalier 1961;
Budd et al. 1996) et d'Aquitaine (100 espèces réparties dans 40 genres au Burdigalien d'après Cahuzac \& Chaix 1993, 1996), aucune structure récifale n’a pu être décelée malgré une biodiversité bien supérieure (faciès subrécifaux à dominante marno-sableuse).

\section{CONCLUSION}

16 espèces récifales appartenant à huit genres coralliens composent la faune des récifs frangeants et côtiers de la région de Bonifacio au Burdigalien supérieur. Les deux associations coralliennes signalent des milieux peu profonds, de mode battu, et ont permis de retracer l'évolution paléoenvironnementale dans cette région. La première concerne les récifs les plus diversifiés (épisode R1), la deuxième appauvrie concerne les récifs les plus étendus (édifices $\mathrm{R} 2$ et R3). L'ensemble de la faune, les morphologies récifales et les organismes associés indiquent des paléoprofondeurs estimées à 5-30 m. Les facteurs paléoenvironnementaux locaux tels que l'exposition à la houle, la forte turbulence et les apports détritiques ont influencé le développement des formes coloniales recouvrantes et ont pu freiner la biodiversité corallienne. Les processus d'édification sont marqués par une zonation récifale discrète, une domination des formes recouvrantes épaisses et la réduction des espèces lors de la période d'intensification maximale de la croissance corallienne (épisodes R2 et R3), avant leur disparition définitive au Langhien inférieur.

\section{Remerciements}

Les auteurs remercient sincèrement Bernard Lathuilière et le rapporteur anonyme pour les corrections du manuscrit. Ce travail a été effectué dans le cadre de la thèse de F. Galloni (Université de Provence), en collaboration avec l'université de Corse et ELP EP puis TOTAL (projet ARTEP). Crédits photographiques L. Cazes (MNHN) et F. Galloni.

\section{RÉFÉRENCES}

ACHIARDI A. D' 1868. - Studio comparativo fra I coralli dei terreni terziari del Piemonte e dell'Alpi venete. Tipografia dei FF. Nistri, Pisa, 58 p., 10 pls.

ARCHIAC A. D' 1869. — Voir TCHIHATCHEFF P. 1866-1869.

ANDRÉ J. P., BARTHET Y., FERRANDInI M., FERRANDini J., REYNAUd J.-Y. \& Tessier B. 2011. - The Bonifacio formation (Miocene of Corsica): transition from a wave-to-tide-dominated coastal system in mixed carbonate-siliciclastic setting. Bulletin de la Société géologique de France, t. 182, n³: 221-230.

Arnaud M., Lơ̈e-Pilot M.-D., Magné J., Monleau C. \& NÉGRETTI B. 1989. - Le Néogène corse. Groupe français d'étude du Néogène, Livret guide excursion, 32 p.

Arnaud M., Magné J., Monleau C., Négretti B. \& Oggiano G. 1992. - Nouvelles données stratigraphiques du Nord-Ouest de la Sardaigne (Italie). Comptes Rendus de l'Académie des Sciences, Paris, t. 315, série II: 965-970.

Assorgia A., Barca S., Casula G. \& Spano C. 1988. - Le successioni sedimentarie e vulcaniche del Miocene nei dintorni di Giave e Cossoine (Logudoro, Sardegna NW). Bollettino della Societa Sarda di Scienze Naturali 26: 75-107. 
BARCA S. \& SPANO C. 2002. - Nuova classidicazione biostratigrafica e geochronologica delle unità formazionali oligoceniche $e$ neogeniche della Sardegna. Correlazioni con le omologhe unità della Corsica. Rendiconti Seminario, Facoltà Scienze Università Cagliari, vol. 72, fasc. 1: 135-147.

BASSANT P. 1999. - The High-Resolution Stratigraphic Architecture and Evolution of the Burdigalian Carbonate-Siliciclastic Sedimentary Systems of the Mut Basin, Turkey. PhD thesis, GeoFocus 3, n¹256, Université de Fribourg, 278 p.

Bassant P., VAn Buchem F. S. P., STRASSER A. \& GörÜr N. 2005. The stratigraphic architecture and evolution of the Burdigalian carbonate-siliciclastic sedimentary systems of the Mut Basin, Turkey. Sedimentary Geology 173: 187-232.

Bosellini F. R. \& Perrin C. 2008. — Estimating Mediterranean Oligocene-Miocene seasurface temperatures: an approach based on coral taxonomic richness. Palaeogeography, Palaeoclimatology, Palaeoecology 258: 71-88.

Brandano M., Tomassetti L., Bosellini F. \& Mazzucchi A. 2010. - Depositional model and paleodepth reconstruction of a coral-rich, mixed siliciclastic-carbonate system: the Burdigalian of Capo Testa (northern Sardinia, Italy). Facies 56: 433-444.

BUCHBINDER B. 1996. - Miocene carbonates of the eastern Mediterranean, the Red Sea and the mesopotamian basin: geodynamic and eustatic controls. Models for carbonate stratigraphy from Miocene reef complexes of Mediterranean regions, SEPM Society for Sedimentary Geology, Concepts in Sedimentology and Paleontology 5: 89-97.

Budd A. F., Bosellini F. R. \& Stemann T. A. 1996. - Systematics of the Oligocene to Miocene reef coral Tarbellastraea in the northern Mediterranean. Palaeontology 39, part. 3: 515-560.

CAHUZAC B. \& CHAIX C. 1993. — Les faunes de coraux (Anthozoaires Scléractiniaires) de la façade atlantique française au Chattien et au Miocène. Proceedings of the 1st R.C.A.N.S. congress, Lisboa, October 1992: 57-69.

CAHUZAC B. \& CHAIX C. 1996. - Structural and faunal evolution of Chattian-Miocene reefs and corals in western France and the northeastern Atlantic ocean. Models for carbonate stratigraphy from Miocene reef complexes of Mediterranean regions, SEPM Society for Sedimentary Geology, Concepts in Sedimentology and Paleontology 5 : 105-127.

Cahuzac B. \& Chaix C. 2009. - Première faune de Scléractiniaires découverte dans l'Aquitanien du Plantat (Saint-Morillon, Gironde, SW France). Contribution à la connaissance de l'étage Aquitanien, $\mathrm{n}^{\circ}$ 2. Bulletin de la Société linnéenne de Bordeaux, 144, nouv. série n ${ }^{\circ} 37$ (1): 25-55.

CARON J. M. \& LOŸE-PILOT M. D. 1990. - Notice explicative de la feuille Pietra-di-Verde à 1/50000. Avec la collaboration de Conchon O., Dominici R., Scius H. \& Vautrelle C. Feuille n ${ }^{\circ} 1115$. Éditions du Bureau de Recherches géologiques et minières, Orléans, $51 \mathrm{p}$.

Carmignani L., Decandia F.A., Disperati L., Fantozzi P.L., Lazzarotto A., Liotta D., Oggiano G. 1995. - Relationships between the Tertiary structural evolution of the SardiniaCorsica-Provençal Domain and the Northern Apennines. Terra Nova 7: 128-137.

ChaIX C. \& CAHUZAC B. 2005. - Les faunes de Scléractiniaires dans les faluns du Miocène moyen d'Atlantique-Est (bassins de la Loire et d'Aquitaine) : paléobiogéographie et évolution climatique. Annales de Paléontologie, Paris, 91, 1 (numéro spécial "faluns "): 33-72, 2 figs, 5 tableaux, 3 pls.

Chaix C. \& SAint MarTin J. P. 2008. — Les faunes de scléractiniaires hermatypiques dans les plates-formes carbonatées méditerranéennes au Miocène supérieur. Geodiversitas 30 (1): 181-209.

Cherchi A., Mancin N., Montadert L., Murru M., Putzu M. T., SChiaVinotTo F. \& VerrubBi V. 2008. - The stratigraphic response to the Oligo-Miocene extension in the western Mediterranean from observations on the Sardinia graben system (Italy). Bulletin de la Société géologique de France, t. 179, n³: 267-287.
Chevalier J. P. 1958. — Aperçu sur les formations coralliennes du Miocène français. Comptes-Rendus des Congrès des Sociétés savantes de Paris et des départements, section Sciences, sous-section Géologie, Colloque sur le Miocène: 111-121.

ChevalieR J.-P. 1961. — Recherche sur les madréporaires et les formations récifales miocènes de la Méditerranée occidentale Atlas paléontologique. Thèse d'état (Paris), Mémoires de la Société Géologique de France, série A, 93, 560 p.

DemarcQ G. \& Perriaux J. (COORdS) 1984. - Néogène. « Synthèse géologique du Sud-Est de la France: Stratigraphie et paléogéographie ". Mémoire du Bureau de Recherches géologiques et minières 125: 470-519.

DUNCAN P. M. 1863. - On the fossil corals of the west indian islands Quaterly Journal of the Geological Society of London, 19: 406458, pls XIV-XVI.

Esper E. J. C. 1797. - Die Pflanzenthiere inbbildungen nach der Natur mit Farben erleuchtet nebst Beschreibungen. Part 7-8: 169-230.

Esteban M. 1996. - An overview of Miocene reefs from Mediterranean areas: general trends and facies models. Models for Carbonate Stratigraphy from Miocene reef complexes of Mediterranean regions. Models for carbonate stratigraphy from Miocene reef complexes of Mediterranean regions, SEPM Society for Sedimentary Geology, Concepts in Sedimentology and Paleontology: 3-53.

Ferrandini M., Ferrandini J., LoŸe-Pilot M.-D., Butterlin J., Cravatte J. \& Janin M.-C. 1998. - Le Miocène du bassin de Saint Florent (Corse) : modalités de la transgression du Burdigalien supérieur et mise en évidence du Serravallien. Geobios 31 (1): 125-137.

Ferrandini M., Galloni F., Babinot J.-F. \& Margerel J.-P. 2002. — La plate-forme burdigalienne de Bonifacio (Corse du Sud): microfaune (foraminiferes, ostracodes) et paléoenvironnements. Revue de micropaléontologie 45: 57-68.

Ferrandini J., Gattacceca J., Ferrandini M., Deino A., Janin M.-C. 2003. - Chronostratigraphie et paléomagnétisme des dépôts oligo-miocènes de Corse: implications géodynamiques pour l'ouverture du bassin liguro-provençal. Bulletin de la Société géologique de France 174: 357-371.

Ferrandini M., Boudagher-Fadel M. K., Ferrandini J., OudET J. \& ANDRÉ J.-P. 2010. - Nouvelles observations sur les Miogypsinidés du Miocène inférieur et moyen de Provence et de Corse (France) et de Sardaigne septentrionale (Italie). Annales de Paléontologie 96: 67-94.

Galloni F., Cornée J.-J., Rebelle M., Ferrandini M. 2001. Sedimentary anatomies of early Miocene coral reefs in South Corsica (France) and South Sardinia (Italy). Géologie méditerranéenne, XXVIII, $n^{\circ}$ 1-2: 73-77.

GALLONI F. 2003. - Organisation sédimentaire et anatomies récifales des systèmes carbonatés à siliciclastiques oligo-miocènes inférieurs de Provence et du bloc corso-sarde. Thèse de doctorat, Université de Provence, Marseille, $255 \mathrm{p}$.

Galloni F., Chaix C. \& Cornée J.-J. 2014. - Architecture and composition of the Upper Burdigalian z-coral buildups of South Corsica (Mediterranean). Comptes Rendus Geoscience 346 (1-2): 45-51.

GALlONi F. \& CORNÉE J.-J. 2014. — Les constructions coralliennes du Burdigalien supérieur de Bonifacio (Corse-du-Sud). Géologie de la France 1: 47-66.

Gattacceca J., Deino A., Rizzo R., Jones D. S., Henry B., BEAUdoIn B. \& VADEBoin F. 2007. - Miocene rotation of Sardinia: New paleomagnetic and geochronological constraints and geodynamic implications. Earth and Planetary Science Letters 258 (3-4): 359-377.

GRAY J.-E. 1847. - An outline of an arrangement of stony corals. Annals and Magazine of natural History 19: 120-128.

Gregory J. W. 1900. - The corals - Jurassic fauna of Cutch. Paleontologia Indica, sér. IX, vol. 2, 195 p., pls 2a à 27. 
Harzhauser M., Kroh A., Mandic O., Piller W. E., Göhlich U., Reuter M. \& Berning B. 2007. - Biogeographic responses to geodynamics: A key study all around the Oligo-Miocene Tethyan Seaway. Zoologischer Anzeiger 246: 241-256.

LOCARD A. 1873. - Sur la faune des terrains tertiaires moyens de la Corse. Bulletin de la Société géologique de France 3, I: 236-241.

Locard A. \& CotTeAu G. 1877. - Description de la faune des terrains tertiaires de la Corse. Editeur, Paris, 374 p., 17 pls.

loÿe-Pilot M. D., Durand-Delga M., Feinberg H., GouriNARD Y. \& MAGNÉ J. 2004. - Les formations burdigaliennes de Corse orientale dans leur cadre géodynamique. Comptes Rendus Géosciences 336: 919-930.

LoŸE-Pilot M. D. \& MAgné J. 1989. — La Plaine orientale, excursion du groupe français d'étude du Néogène en Corse, livret-guide 4/8 septembre 1989, in ARNAUd M., LOŸE-Pilot M.-D., MAGNÉ J., Monleau C. et Negretti B. (EDS), Le Néogène corse. Groupe français d'études du Néogène, Livret guide d'excursion, $32 \mathrm{p}$.

Martini I. P., Oggiano G. \& Mazzei R. 1992. — Siliciclastic-carbonate sequence of Miocene grabens of Northern Sardinia, Western Mediterranean Sea. Sedimentary Geology 36: 63-78.

MAUREL-FERRANDINI M. 1975. - Reconstitution paléogéographique du Burdigalien du littoral de la chaîne de la Nerthe et de la région des étangs (Bouches du Rhône, France). Thèse, Université AixMarseille I, $87 \mathrm{p}$.

Mazzei R. \& OgGiano G. 1990. - Messa in evidenza di due cicli sedimentari nel Miocene dell'area di Florinas (Sardegna settentrionale). Atti della Societa Toscana di Scienze Naturali, Serie A, 97: 119-147.

MiCHELIN J. L. H. 1840-1847. - Iconographie zoophytologique. Description par localités et terrains des polypiers fossiles de France, et pays environnants. Bertrand eds, Paris, 348 p., 79 pls.

MichelOTTi G. 1838. — Specimen zoophytologiae diluvianae, Augusta Taurinensis. Turin, $227 \mathrm{p}$.

Milne-Edwards H. \& Haime J. 1848-1851. — Recherches sur la structure et la classification des Polypiers récents et fossiles. (vol. 16: 1851a: Poritidae). Annales des Sciences naturelles, série 3, 16: 21-70.

Milne-Edwards H. \& Haime J. 1851b. - Monographie des polypiers fossiles des terrains paléozoïques. Archives du Muséum d'Histoire naturelle, Paris, 5: 1-200 + 201-502, 20 pls.

Milne-Edwards H. \& Haime J. 1857-1860. — Histoire naturelle des coralliaires ou polypes proprement dits. Paris, t. 1 (1857: VIII + 1-95), 2 (1857: 1-268), 3 (1860: 1-219), atlas (1857: 31 pls).

Monleau C., Arnaud M., Magné J., Negretti B. \& Rossi F. 1996. - Précisions stratigraphiques et paléoécologiques sur le Miocène inférieur et moyen du sud de la Corse et du nord de la Sardaigne. Géologie méditerranéenne, t. XXIII, n³-4: 235-241.

D'OrbignY A. C. V. D. 1849-1852. - Prodrome de paléontologie stratigraphique universelle des animaux mollusques et rayonnés, faisant suite au cours élémentaire de paléontologie. Paris, 3 vols.

Orsini J.-B., Ferrandini J., Ferrandini M., Loye M.-D., PluQUeT F., Guennoc P., ThinON I., SANTIAGO M., ReYNAUD J.-Y., Oggiano G., Cherchi G., Gattacceca J., Orru P., Puliga G., Pintus M. \& Ulzega A. (sous presse). — Carte géologique France (1/50 000), feuille Sotta-Bonifacio-Santa-Teresa-di-Gallura (CA1 127). BRGM, Orléans. Notice explicative par : Orsini J.-B., Capdevila R., Ferrandini M., Ferrandini J., Galloni F., André J.-P., Münch P., Saint-Martin S., Alamy Z., Chiari J.-T., Loÿe M.-D., Pluquet F., Guennoc P., Thinon I., Santiago M., Teissier B., Reynaud J.-Y., Gamisans J., Tramoni P., Oggiano G., Cherchi G, Orrù P., Puliga G., Pintus M. \& Ulzega A. (2010), 258 p.
OrsZag-SPerber F. 1978. - Le Néogène de la Corse et ses relations avec la géodynamique de la Méditerranée occidentale. Thèse d'état, Paris-Sud, 328 p.

Orszag-Sperber F. \& Pilot M.-D. 1976. - Grands traits du Néogène de Corse. Bulletin de la Société Géologique de France 7 , t. XVIII, n ${ }^{\circ}$ : 1183-1187.

OSASCO E. 1897. - Di alcuni corallari miocenici del Piemonte. Atti della Reale Accademia delle Scienze di Torino 32: 436-449.

OUDET J. 2008. - Étude terre-mer de la transition syn-rift/post-rift sur les marges de l'océan Liguro-Provençal: apports de la modélisation géologique $3 \mathrm{D}$ et de la chronostratigraphie intégrée. Thèse de doctorat, Université de Provence, Marseille, 288 p.

Oudet J., Munch P., Borgomano J., Quillévéré F., MelinteDobrinescu M., Demory F., Viseur S. \& Cornée J. J. 2010. Land and sea study of the northeastern golfe du Lion rifted margin: the Oligocene-Miocene of southern Provence (Nerthe area, SE France). Bulletin de la Société géologique de France 181: 591-607.

Pedley M. 1996. - Miocene reef distribution and their associations in the central Mediterranean region: an overview. Models for Carbonate Stratigraphy from Miocene reef complexes of Mediterranean regions. Models for carbonate stratigraphy from Miocene reef complexes of Mediterranean regions, SEPM Society for Sedimentary Geology, Concepts in Sedimentology and Paleontology: 73-87.

PERON A. 1886. - Description du terrain tertiaire du sud de l'île de Corse - Comptes rendus Association française pour l'avancement des sciences: 383-409, 7 figs, 1 carte.

Perrin C. \& Bosellini F. R. 2012. - Paleobiogeography of scleractinian reef corals: Changing patterns during the OligoceneMiocene climatic transition in the Mediterranean. Earth-Science Reviews 111: 1-24.

Reynaud J.-Y., Ferrandini M., Ferrandini J., Santiago M., ThiNON I., ANDRÉ J.-P., BARTHET Y., GUENNOC P. \& TESSIER B. 2013. - From non-tidal shelf to tide-dominated strait: The Miocene Bonifacio Basin, Southern Corsica. Sedimentology 60: 599-623.

SISMONDA E. 1871. - Matériaux pour servir à la paléontologie du terrain tertiaire du Piémont. Memorie della Accademia reale della Scienze di Torino, série 2, t. 25: 257-361, pls 1-10.

TChinatchefF P. DE 1866-1869. - Asie Mineure - Paléontologie, par d'Archiac, Fischer et Verneuil (coraux par d'Archiac, atlas pls XIV à XVI). Guérin, Paris, $422 \mathrm{p}$.

TomassetTi L. \& BRANDANO M. 2013. - Sea level changes recorded in mixed siliciclastic-carbonate shallow-water deposits: The Cala di Labra Formation (Burdigalian, Corsica). Sedimentary Geology 294: 58-67.

Tomassetti L., Bosellini F. \& BRAndano M. 2013. - Growth and demise of a Burdigalian coral bioconstruction on a granite rocky substrate (Bonifacio Basin, southeastern Corsica). Facies 59: 703-716

VAUGHAN T. W. 1919. - Fossils Corals from Central America, Cuba and Porto-Rico, with an Account of the American Tertiary, Pleistocene and Recent Coral Reefs. Bulletin of the United States National Museum, vol. 103. United States Government Publishing Office, Washington: 189-524, pls 68-152.

Veron J. E. N. 2000. - Corals of the World. Australian Institute of Marine Science, vol. 1: 463 p.; vol. 2: 429 p.; vol. 3: 490 p.

ZufFardi-CommerCi R. 1932. - Corallari-Zoantari fossili del Miocene della "Collina di Torino". Palaeontographia Italica 33: 85-132.
Soumis le 10 avril 2014; accepté le 22 janvier 2015; publié le 25 septembre 2015. 
